\title{
Modulation of the electronic properties of GaN films by surface acoustic waves
}

J. Camacho ${ }^{\text {a) }}$

Departamento Física Aplicada, Universidad Politécnica de Cartagena, Campus Muralla del Mar, E-30202 Cartagena (Murcia), Spain

P. V. Santos, F. Alsina, M. Ramsteiner, and K. H. Ploog

Paul-Drude-Institut für Festkörperelektronik, Hausvogteiplatz 5-7, 10117 Berlin, Germany

\section{A. Cantarero}

Materials Science Institute, Universitat de València, P.O. Box 22085, E-46071 València, Spain

H. Obloh and J. Wagner

Fraunhofer-Institut für Angewandte Festkörperphysik, Tullastrasse 72, 79108 Freiburg, Germany

(Received 28 March 2003; accepted 24 April 2003)

\begin{abstract}
We report on the interaction between photogenerated electron-hole pairs and surface acoustic waves (SAW) in GaN films grown on sapphire substrates. The spatial separation of photogenerated carriers by the piezoelectric field of the SAW is evidenced by the quenching of the photoluminescence (PL) intensity. The quenching levels in $\mathrm{GaN}$ are significantly smaller than those measured in GaAs under similar conditions. The latter is attributed to the lower exciton ionization efficiency and carrier separation probabilities mediated by the piezoelectric effect. The PL spectra also evidence energy shifts and broadenings of the electronic transitions, which are attributed to the band gap modulation by the SAW strain field. (C) 2003 American Institute of Physics. [DOI: 10.1063/1.1582556]
\end{abstract}

\section{INTRODUCTION}

Developments in the epitaxial growth of GaN-based films during recent years have opened additional areas of application of these films in electronic and optoelectronic devices. ${ }^{1}$ The large band gap of GaN has been successfully used to realize optoelectronic devices such as lasers and light-emitting diodes for the ultraviolet energy range. The large gap is also advantageous for high-power applications and electronic devices operating at high temperatures.

The stronger piezoelectric coupling coefficients arising from the larger ionicity as well as the higher acoustic velocities as compared to $(\mathrm{Al}, \mathrm{Ga}) \mathrm{As}$ materials make $\mathrm{GaN}$ and $\mathrm{AlN}$ films interesting for surface acoustic wave (SAW) devices based on semiconductors. ${ }^{2-4}$ SAW applications of GaNbased materials include, besides high-frequency electronic filters for telecommunication, ${ }^{4}$ electro- and acousto-optic light modulators for the ultraviolet spectral range. ${ }^{5,6} \mathrm{~A}$ further advantage of $(\mathrm{Al}, \mathrm{Ga}) \mathrm{N}$-based films resides on the wider tunability of the elastic properties with film composition as compared to the (Al,Ga)As system. The latter can be employed to control the depth distribution of the acoustic field and to manipulate the SAW modes using acoustic waveguides. ${ }^{4}$

The modulation of the band edges by the SAW piezoelectric field provides an efficient way of controlling the electronic properties of semiconductor structures. The piezoelectric field induces a type-II lateral modulation of the band edges, which strongly interacts with charge carriers. In GaAs-based materials, this modulation has been applied to

\footnotetext{
a)Electronic mail: jcamacho@upct.es
}

store and transport single carriers ${ }^{7}$ as well as photogenerated electron-hole (e-h) pairs. ${ }^{8,9}$ The latter is based on the ionization of photogenerated $\mathrm{e}-\mathrm{h}$ pairs and subsequent trapping of the free electrons and holes in the maxima and minima of the piezoelectric potential, which propagates with the SAW velocity $v_{\text {SAW }}$. The acoustically induced transport requires that the drift velocity $v_{c}=\left.\mu F_{x}\right|_{\max }$ ( $\mu$ denotes the carrier mobility) induced by the longitudinal component $F_{x}$ of the piezoelectric field exceeds $v_{\text {SAW }}$ for both electrons and holes. The spatial separation considerably increases the recombination lifetimes, thus providing a mechanism for the storage of $\mathrm{e}-\mathrm{h}$ pairs.

In this article, we investigate the interaction of photogenerated carriers with SAWs in GaN films using spatially resolved photoluminescence (PL) spectroscopy. As demonstrated for GaAs-based SAW structures, ${ }^{8-10}$ the piezoelectric field leads to a suppression of the PL due to the spatial separation of electrons and holes. The degree of suppression, however, is substantially lower than in GaAs, a result attributed to the reduced exciton ionization probability and the lower carrier mobility in GaN. In addition to the reduction in oscillator strength, the PL lines shift in energy and broaden under a SAW. We demonstrate that the last effect arises from the modulation of the band edges by the SAW strain field.

\section{EXPERIMENT}

The film under investigation consists of a $2.5-\mu \mathrm{m}$-thick semi-insulating $\mathrm{GaN}$ layer grown by metalorganic vaporphase epitaxy on a sapphire substrate. The $c=[0001]$ axis of the GaN film and of the substrate are parallel and oriented 
along the growth direction. The in-plane $a \equiv x$ and $b \mathrm{GaN}$ axes, in contrast, are rotated by $30^{\circ}$ with respect to those of the substrate.

The optical experiments were performed on SAW-delay lines consisting of two split-finger interdigital transducers (IDTs) deposited on the surface of the GaN films. The high sheet resistance of the layers $(>10 \mathrm{k} \Omega)$ is important for the SAW generation since it avoids the screening of the electric fields created by the IDTs. Under rf excitation, the IDTs launch Rayleigh-like SAWs with a wavelength $\lambda_{\text {SAW }}$ $=5.6 \mu \mathrm{m}$ (corresponding to a frequency of $745.7 \mathrm{MHz}$ at room temperature and of $748.2 \mathrm{MHz}$ at $6 \mathrm{~K}$ ) along the $x$ $=[1 \overline{1} 00]$ direction of the substrate. The finger area of the split-finger IDTs are $1960 \mu \mathrm{m}$ long and $120 \mu \mathrm{m}$ wide. The thickness and width of the individual aluminum fingers are $60 \mathrm{~nm}$ and $0.7 \mu \mathrm{m}$, respectively. The employed finger width $l_{f}$ corresponds to the minimum line width of the lithographic process used for IDT fabrication. The latter determines the SAW wavelength, which is equal to $8 l_{f}=5.6 \mu \mathrm{m}$ for splitfinger IDTs. The amplitude of the SAW will be specified in terms of the nominal rf power $P_{\text {rf }}$ applied to the IDT, which does not include effects associated with rf coupling losses. By measuring the rf reflection using a network analyzer, we determine that only $\eta \sim 15 \%$ of the input electrical power is converted into surface acoustic modes at $6 \mathrm{~K}$.

The SAW beam induces a periodic modulation of the surface, which was probed using a confocal microscope operating in a Michelson interferometer configuration. The interferometric measurements were performed at room temperature with a spatial resolution of approximately $1 \mu \mathrm{m}$. A frequency-doubled Nd-vanadate laser $\left(\lambda_{L}=532 \mathrm{~nm}\right)$ was employed as light excitation source. These measurements allow us to determine the absolute linear power density $P_{l}$ of the SAW beam, defined as the total acoustic power flow per unit length perpendicular to the beam cross section.

For the PL studies, the samples were mounted in a liquid-helium microscope cryostat $(T \approx 6 \quad \mathrm{~K})$ with $\mathrm{rf}$ feedthroughs for SAW excitation. The PL was excited using the $\lambda_{\mathrm{L}}=325 \mathrm{~nm}$ line of a $\mathrm{He}-\mathrm{Cd}$ laser (laser intensity of approximately $5 \mu \mathrm{W}$ ) focused to a micrometer-sized spot using a $10 \times$ microscope objective. The PL light was collected by the same objective and analyzed by a Jobin Yvon spectrograph equipped with a cooled charge-coupled-device detector. The setup has also capabilities to record spatially resolved PL profiles by scanning the laser spot relative to the sample.

The PL studies on GaN require the application of high rf powers (up to $24 \mathrm{~dB}$ ) to the IDTs to generate large SAW fields. The large powers may induce temperature changes, which partially mask the changes in the PL spectrum induced by the SAW fields. In addition, the SAW field may induce carrier trapping by surface states, which distorts the potential distribution. ${ }^{11,12}$ In order to eliminate these effects, which take place on a time scale much longer than the SAW period, differential PL measurements were performed by chopping the exciting laser beam and the rf power applied to the IDT at the same frequency of $630 \mathrm{~Hz}$. In these experiments, which will be denoted as chopped (ch) in contrast to the

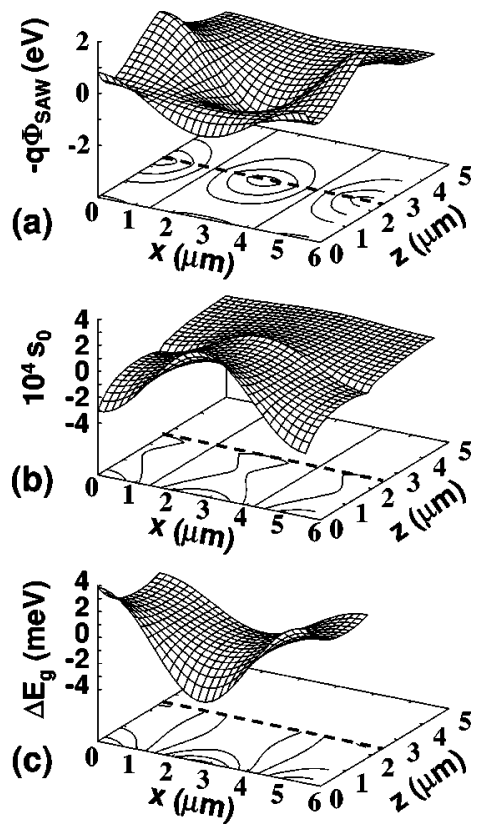

FIG. 1. Profiles for the (a) piezoelectric potential energy $-q \Phi_{\mathrm{SAW}}$, (b) hydrostatic strain $s_{0}$, and (c) strain contribution to the band gap modulation induced by a SAW in a $2.5-\mu \mathrm{m}$-thick GaN layer on a sapphire substrate. The SAW propagates along the $x=[1 \overline{1} 00]$ axis of the sapphire substrate, the $z$ axis is oriented along the growth direction. The dashed line indicates the interface between the GaN film and the sapphire substrate. The calculations were performed for $P_{\mathrm{rf}}=22 \mathrm{~dB}$, which corresponds to a linear power density of $P_{l}=216 \mathrm{~W} / \mathrm{m}$.

continuous (cw) illumination measurements, the direct effect of the SAW on the carriers appears when the laser and the rf power are chopped in phase. When the two excitations are out of phase (i.e., the laser illuminates the sample when no rf power is applied to the IDT), one reproduces the conditions in the absence of a SAW. This procedure, which allows for the separation of effects induced by the fast SAW fields from slower effects induced by heating, becomes particularly important for the determination of the small transition energy shifts induced by the SAW field, as will be discussed in detail is Sec. III C.

\section{RESULTS}

\section{A. SAW fields}

Figures 1(a) and 1(b) display profiles for the electronic potential energy $-q \Phi_{\text {SAW }}$ induced by the piezoelectric field and for the hydrostatic strain $s_{0}=\epsilon_{x x}+\epsilon_{y y}+\epsilon_{z z}$, respectively, calculated for the sample structure described earlier. In the last expression, $\epsilon_{i i}$ denotes the strain components along the $x=[1 \overline{1} 00], y=[11 \overline{2} 0]$, and $z=[0001]$ directions of the sapphire substrate. The SAW propagates along the sapphire $x$ axis with a linear power density of $216 \mathrm{~W} / \mathrm{m}$ (corresponding to the one obtained in the experiments when the IDTs are excited with $P_{\mathrm{rf}}=22 \mathrm{~dB}$ ). The calculations were carried out by solving the elastic equations for SAW propagation in the GaN/sapphire structure using the elastic data for the GaN film reported in Ref. 3. The fundamental Rayleigh mode with wavelength $\lambda_{\mathrm{SAW}}=5.6 \mu \mathrm{m}$ is found to have a calculated frequency of $f_{\mathrm{SAW}}=743.342 \mathrm{MHz}$, which com- 


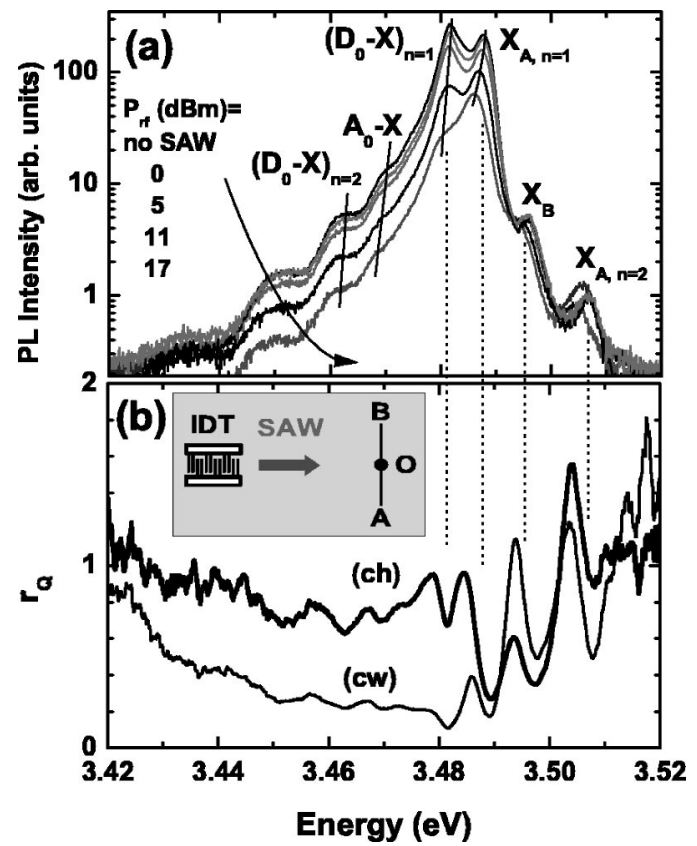

FIG. 2. (a) Photoluminescence spectra of a GaN film on sapphire recorded in the absence of a SAW (upper curve) and for power levels $P_{\mathrm{rf}}$ applied to the IDT ranging from $0 \mathrm{~dB}$ (upper curve) to $17 \mathrm{~dB}$ (lower curve). The assignment of the electronic transitions follows Refs. 14 and 16. (b) Quenching ratio $r_{\mathrm{Q}}$ measured under the $\mathrm{cw}$ and ch illumination procedures for a rf power $P_{\mathrm{rf}}=17 \mathrm{~dB}$. The PL measurements were performed at the point $O$ indicated in the inset of (b), which is located on the SAW path approximately $1 \mathrm{~mm}$ away from the IDT.

pares well with the experimental value of $745.7 \mathrm{MHz}$ measured at room temperature. Note that although the SAW wavelength is more than twice as large as the GaN thickness, the higher SAW velocity of the sapphire substrate leads to a strong confinement of the acoustic field within the $\mathrm{GaN}$ layer. The piezoelectric potential energy, in contrast, is stronger around the GaN/sapphire interface. Note that the piezoelectric potential in Fig. 1(a) represents only the SAW contribution and does not include the effects of the spontaneous polarization and static strains in the GaN films. ${ }^{13}$

\section{B. PL quenching}

The PL spectrum of the GaN films recorded in the absence of a SAW is dominated by the emission lines from free and bound excitons, as illustrated by the upper curve in Fig. 2(a). This spectrum was recorded for $\mathrm{cw}$ laser excitation at the center of the SAW beam, at a point $O$ located approximately $1 \mathrm{~mm}$ away from the IDT [see inset in Fig. 2(b)]. The spectral features were denoted following the assignment in Refs. 14-16. The main features correspond to the emission from the free excitons $X_{A, n=1,2}$ and $X_{B}$ and from the exciton bound to a neutral donor $\left(D_{0}-X\right)$. Weaker lines corresponding to excitons bound to acceptors and to phonon replicas are observed at lower energies.

Under the influence of a SAW, the PL lines redshift while their intensities decrease, as illustrated by additional curves for increasing rf powers in Fig. 2(a). The behavior is not shared by the $X_{A, n=2}$ : although it redshifts, the intensity of this line increases with SAW intensity. Figure 2(b) shows the quenching ratio $r_{Q}$, defined as the ratio between the PL intensity in the presence and in the absence of a SAW. The curves marked by $\mathrm{cw}$ and ch were obtained under continuous and under chopped rf excitation, respectively. In the latter case, slow mechanisms for the quenching (such as those induced by sample heating) are eliminated, which reduce the overall quenching ratios $r_{Q}$ under continuous light and $\mathrm{rf}$ excitations. The ch curve reflects the direct interaction of the SAW with carriers, i.e., the spatial separation of the e-h pairs by the piezoelectric field of the SAW. ${ }^{8-10}$ We checked that $r_{Q}$ is independent of the chopping frequency within the probed range from 300 to $650 \mathrm{~Hz}$.

The degree of PL quenching increases with the intensity of the SAW beam and with decreasing intensity of the excitation light. In general, however, the overall quenching ratios (integrated over the energy range of the PL signal) in the GaN films are considerably smaller than in GaAs measured under comparable conditions, where it can be as low as $10^{-2}{ }^{17}$ The small quenching effect is attributed to two mechanisms: the large exciton binding energy, which hinders the ionization of photogenerated $\mathrm{e}-\mathrm{h}$ pairs, and the low mobility of the carriers, which prevents the spatial separation of electrons and holes by the SAW field. The calculations of the SAW fields of Sec. III A yield a maximum value $F_{x}=10^{4}$ $\mathrm{V} / \mathrm{cm}$ for the longitudinal piezoelectric field near the surface, where most of the incoming light is absorbed. This field was obtained for a SAW with linear power density of $216 \mathrm{~W} / \mathrm{m}$, which corresponds to the maximum power applied to the IDT in the present experiments $\left(P_{\mathrm{rf}}=22 \mathrm{~dB}\right)$. The direct exciton ionization through the tunneling mechanism ${ }^{18}$ requires much higher fields (of the order $E_{B} / a_{\text {Bohr }} \sim 8 \times 10^{4} \mathrm{~V} / \mathrm{cm}$, where $E_{B}=26 \mathrm{meV}$ denotes the exciton binding energy and $a_{\text {Bohr }}=3 \mathrm{~nm}$ the excitonic radius). The excitons, however, can also be ionized through impact with carriers, a process which requires significantly weaker fields. ${ }^{9,19}$ The anomalous behavior of the $X_{A, n=2}$, whose intensity increases with the SAW amplitude, is probably due to the capture of carriers produced during the ionization of lower lying excitonic states. Such a behavior has been reported for exciton ionization experiments using static fields in GaN. ${ }^{19}$

Carrier separation is expected to be limited by the hole mobility, which in $\mathrm{GaN}$ is approximately an order of magnitude lower than that of electrons. ${ }^{20}$ From the measured SAW velocity $v_{\text {SAW }}=4180 \mathrm{~m} / \mathrm{s}$, we estimated a lower limit for the hole mobility required for SAW-induced transport of $v_{\text {SAW }} / F_{x}=40 \mathrm{~cm}^{2} /(\mathrm{V} \mathrm{s})$. Hole mobilities of the order of 100 $\mathrm{cm}^{2} /(\mathrm{V} \mathrm{s})$ have been reported for undoped $\mathrm{GaN}$ films at room temperature. ${ }^{20}$ Hole mobility data are not available for undoped $\mathrm{GaN}$ for temperatures below $250 \mathrm{~K}$ to allow for a comparison with the present data. As for the electron mobility, the hole mobility is expected to increase with decreasing temperature down to approximately $100 \mathrm{~K}$, but then to reduce significantly for lower temperatures due to the capture of the carriers by localized states.

The PL quenching can be used to map the spatial distribution of the piezoelectric field across the width of the SAW beam. Figures 3(a) and 3(b) display the amplitude of the vertical surface displacement $u_{z}$ measured by interferometry and the integrated PL intensity, respectively, recorded along 


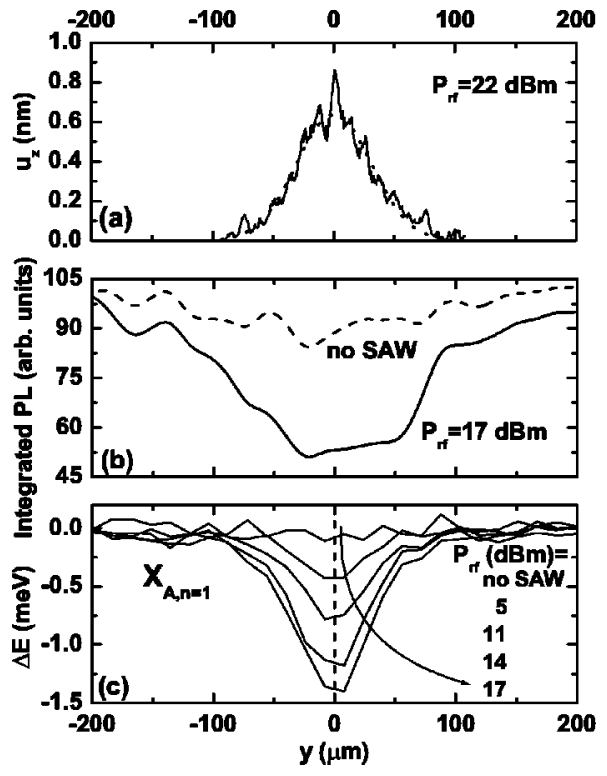

FIG. 3. (a) Vertical displacement $u_{z}$ measured by interferometry along the line $A-B$ indicated in the inset of Fig. 2(a). The interferometric measurements were performed at room temperature by exciting the IDT with $P_{\mathrm{rf}}$ $=22 \mathrm{~dB}$. The dashed line is a Gaussian fit to the experimental data. (b) Spatial profiles of the integrated PL response (integration range from 3.47 to $3.50 \mathrm{eV}$ ) recorded at low temperatures along the same line in the presence (thick line, $P_{\mathrm{rf}}=17 \mathrm{~dB}$ ) and in the absence of a SAW (dashed line). (c) Energy shifts of the $X_{A, n=1}$ excitonic line induced by SAW with different amplitudes.

the line $A-B$ indicated in the inset of Fig. 2(b). The PL was integrated over the energy range from 3.47 to $3.50 \mathrm{eV}$. The SAW beam has a Gaussian-like cross section with a full width at half maximum of $70 \mu \mathrm{m}$ at a position $1 \mathrm{~mm}$ away from the IDT. The latter corresponds to approximately half of the IDT aperture of $120 \mu \mathrm{m}$. The small structures in the PL profile reflect the inhomogeneity in the PL response along the scan line, which is also present in the absence of a SAW (dashed line). As for the interferometric data in Fig. 3(a), the PL quenching is only observed within the SAW beam width. The apparent beam width obtained from the PL measurements is larger than that expected from interferometry. The difference is attributed to the distortion of the profiles induced by the nonlinear dependence of the PL intensity on the SAW amplitude. For quenching ratios $1<r_{Q}<0.3$, the PL intensity decreases logarithmically with SAW power.

\section{Energy shifts}

Further information about the interaction between the SAW and the materials band structure is obtained from the energy and from the linewidth of the spectral PL features. As indicated in Fig. 2(a), the PL lines shift to lower energies with increasing SAW amplitudes, leading to the derivativelike spectral shape of $r_{Q}$ in Fig. 2(b). In order to obtain reliable values for the energy shifts and for the changes in linewidths induced by the SAW, the PL spectra measured using the modulation procedure described in Sec. II were fitted with a sum of Gaussian curves used to represent the different transitions in Fig. 2(a). For each SAW intensity, the shifts $\Delta E$ were corrected for temperature changes by sub-

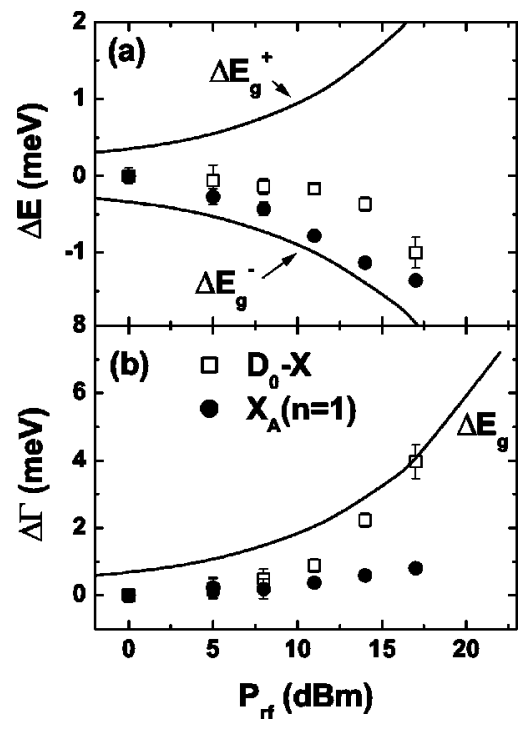

FIG. 4. Changes in (a) energy $\Delta E$ and (b) linewidth $\Gamma$ of the PL line from the $X_{A, n=1}$ (dots) and $D_{0}-X$ (squares) transitions as a function of the SAW rf power $P_{\text {rf }}$. The lines in (a) display the calculated maximum $\left(\Delta E_{g}^{+}\right)$and minimum $\left(\Delta E_{g}^{-}\right)$energy shifts induced by the SAW strain near the sample surface. The line in (b) shows the peak-to-peak modulation of the band gap $\Delta E_{g}=\Delta E_{g}^{+}-\Delta E_{g}^{-}$.

tracting the corresponding shifts measured when the light and rf power are chopped out of phase. This differential procedure allows for the determination of energetic shifts much smaller than the characteristic width of the PL lines. An example is illustrated in Fig. 3(c), which displays the spatial dependence of $\Delta E$ across the SAW beam. Note that the profiles for the absolute values of $\Delta E$ follow closely the spatial dependence of the vertical displacement $u_{z}$ in Fig. 3(a).

Figures 4(a) and 4(b) display the variations in the mean energy $\Delta E$ and in the linewidth $\Delta \Gamma$ of the main lines $X_{A, n=1}$ (dots) and $D_{0}-X$ (squares) induced by a SAW as a function of the rf power applied to the IDT. Although both excitonic lines are associated with the same band edge states, they display qualitatively different dependencies on the amplitude of the SAW field. While the line width of the $X_{A, n=1}$ line does not vary appreciably as the line redshifts, the $D_{0}-X$ line broadens considerably for $P_{\mathrm{rf}}<15 \mathrm{~dB}$, without substantially changing its center position. As will be demonstrated below, the different behaviors are associated with the extended and localized character of the $X_{A, n=1}$ and $D_{0}-X$ lines, respectively.

The energetic changes of the PL lines are associated with the band gap modulation induced by the strain and by the piezoelectric field. The energy shifts induced by the hydrostatic strain component increase linearly with the SAW amplitude and are expected to be the dominating mechanism for small SAW intensities. Energy shifts associated with the piezoelectric field, which are quadratic in the SAW amplitude, are likely to appear only for high SAW intensities. We checked for contributions from the piezoelectric field by performing PL measurements with different excitation intensities. The high carrier density produced by large photon fluxes screens the piezoelectric field, thus leading to lower PL quenching ratios. At the same time, energy shifts induced by 
the electro-optical effect are expected to decrease. We measured, however, similar energy shifts over the whole range of intensities investigated here (from 0.5 to $50 \mu \mathrm{W}$ ), thus indicating that the piezoelectric field has a minor influence on the energetic position of the PL line.

The sinusoidal band gap modulation induced by the strain field splits the PL line into a pair of lines shifted by $\Delta E_{g}^{+}$and $\Delta E_{g}^{-}$with respect to the transition energy in the absence of a SAW. The energetic separation between the doublet lines $\Delta E_{g}=\Delta E_{g}^{+}-\Delta E_{g}^{-}$corresponds to the difference between the minimum and maximum gaps. ${ }^{17,21}$ The splitting into two lines, instead of a simple broadening, is associated with the large time-averaged joint density of states for transitions between the conduction and valence bands at the positions of minimum and maximum band gaps induced by the sinusoidal modulation. ${ }^{17} \mathrm{~A}$ clear splitting is not observed in the present experiments because the PL linewidth is larger than $\Delta E_{g}$. As will become clear in the following paragraphs, the ratio between the oscillator strengths of the doublet components also depends on the SAW amplitude, which leads to an energetic shift of the measured PL line.

The strain-induced shifts of the conduction and valence bands were calculated by diagonalizing the strain Hamiltonian by Bir and Pikus ${ }^{22}$ for the hexagonal GaN crystal. The calculations were performed by using the full strain tensor determined by the elastic calculations of the SAW fields. The deformation potentials required to calculate the energy shifts from the strain field were extracted from Refs. 23 and 24. The spatial dependence of the gap modulation $\Delta E_{g}$ is displayed in Fig. 1(c). The latter is mainly determined by the volume changes induced by the hydrostatic component of the strain field, which is shown in Fig. 1(b). The largest band gap modulation is found near the surface of the sample. The energy shifts of the high $\left(\Delta E_{g}^{+}\right)$and low $\left(\Delta E_{g}^{-}\right)$strain-splitted PL components at the surface are displayed as a function of the nominal rf power by the solid lines in Fig. 4(a).

The energy shifts of the $X_{A, n=1}$ line in Fig. 4(a) follow reasonably well the calculated energy of the $\Delta E_{g}^{-}$transition near the surface. In fact, due to the strong absorption of the excitation light, most of the $\mathrm{e}-\mathrm{h}$ pairs are expected to be generated near the sample surface. In contrast, the oscillator strength of the $\Delta E_{g}^{+}$transition appears to be very weak, since the linewidth of the $X_{A, n=1}$ transition remains small over the whole range of SAW amplitudes. In order to understand this difference in oscillator strength between the $\Delta E_{g}^{+}$and $\Delta E_{g}^{-}$ doublet components, we have to consider the spatial distribution of electron and holes in the SAW potential. Due to their higher mobility, electrons can easily follow the dynamic piezoelectric potential: these carriers become concentrated at the positions of minimum energy $-q \Phi_{\mathrm{SAW}}$ [see Fig. 1(a)]. Holes, on the other hand, are much less mobile and have a wider spatial distribution in the SAW potential. The spatial distribution of the PL becomes, therefore, determined by the electron concentration. According to Fig. 1, the phase relationship between the strain and piezoelectric potential is such that electrons drift towards the regions of minimum potential energy and band gap. Preferential recombination is thus expected to take place in these regions, in agreement with Fig. 4(a).
In contrast to the $X_{A, n=1}$ line, the $D_{0}-X$ line broadens considerably with the SAW amplitude while its average energy only shifts for very high SAW powers. This behavior is attributed to the large electron capture cross section of ionized donors, which traps photogenerated electrons before they can drift over distances of the order of $\lambda_{\mathrm{SAW}} / 2$ in the piezoelectric field. Since the spatial modulation of the hole concentration is weak, bound $D_{0}-X$ excitons form with approximately the same probability at the positions of small and large band gap. As a result, the effective PL linewidth increases with $\Delta E_{g}$, while the average PL energy remains approximately constant. This behavior is in agreement with the experimental data in Fig. 4.

\section{CONCLUSIONS}

In conclusion, we have investigated the interaction between photogenerated carriers and the acoustic and piezoelectric fields induced by a SAW in GaN using spatially resolved PL. The spatial separation of photogenerated carriers by the piezoelectric field is evidenced by a quenching of the PL intensity. The PL quenching effect in GaN is significantly smaller than in GaAs, as expected from the lower exciton ionization efficiency and carrier mobility. From the values of the piezoelectric field, we estimated an upper limit of approximately $40 \mathrm{~cm}^{2} /(\mathrm{V} \mathrm{s})$ for the hole mobility in intrinsic $\mathrm{GaN}$ at low temperatures. As far as the acoustically induced transport is concerned, these results show that the stronger piezoelectric fields expected from the higher piezoelectric coupling of $\mathrm{GaN}$ as compared to GaAs do not compensate for the lower mobility of the carriers.

The PL spectra also reveal a shift and broadening of the electronic transition energy. By comparison with band structure calculations, we demonstrate that these effects are associated with the modulation of the band edges by the SAW strain field. The dependence of these quantities on SAW intensity is considerably different for localized and for free excitons. The latter is attributed to the different exciton formation dynamics in the two cases.

\section{ACKNOWLEDGMENTS}

The authors thank H. Grahn for comments and for a critical reading of the manuscript, as well as W. Seidel and Krauß for the fabrication of the SAW transducers. They also acknowledge the partial financial support from the Ministry of Science of Technology of Spain and the DAAD/Germany.

${ }^{1}$ M. Tchounkeu, O. Briot, B. Gil, J. P. Alexis, and R.-L. Aulombard, J. Appl. Phys. 80, 5352 (1996).

${ }^{2}$ H. Okano, N. Tanaka, Y. Takahashi, T. Tanaka, K. Shibata, and S. Nakano, Appl. Phys. Lett. 64, 166 (1994).

${ }^{3}$ C. Deger, E. Born, H. Angerer, O. Ambacher, M. Stutzmann, J. Hornsteiner, E. Riha, and G. Fischerauer, Appl. Phys. Lett. 72, 2400 (1998).

${ }^{4}$ Y. Takagaki, P. V. Santos, E. Wiebicke, O. Brandt, and H.-P. Schönherr, Appl. Phys. Lett. 81, 2538 (2002).

${ }^{5}$ R. Rimeika, D. Ciplys, R. Gaska, J. W. Yang, M. A. Khan, M. S. Shur, and E. Towe, Appl. Phys. Lett. 77, 480 (2000).

${ }^{6}$ D. Ciplys, R. Gaska, M. Shur, R. Rimeika, J. W. Yang, and A. Khan, in Materials and Devices for Photonic Circuits II, edited by M. N. Armenise (SPIE, Bellingham, WA, 2001), Vol. 4453, pp. 37-44.

${ }^{7}$ M. J. Hoskins, H. Morkoç, and B. J. Hinsinger, Appl. Phys. Lett. 41, 332 (1982). 
${ }^{8}$ C. Rocke, S. Zimmermann, A. Wixforth, J. P. Kotthaus, G. Böhm, and G. Weimann, Phys. Rev. Lett. 78, 4099 (1997).

${ }^{9}$ P. V. Santos, M. Ramsteiner, and F. Jungnickel, Appl. Phys. Lett. 72, 2099 (1998).

${ }^{10}$ K. S. Zhuravlev, D. P. Petrov, Y. B. Bolkhovityanov, and N. S. Rudaja, Appl. Phys. Lett. 70, 3389 (1997).

${ }^{11}$ N. J. Moll, O. W. Otto, and C. F. Quate, J. Phys. Colloq. 33, 231 (1972).

${ }^{12}$ I. J. Fritz and T. M. Brennan, Semicond. Sci. Technol. 12, 19 (1997).

${ }^{13}$ O. Ambacher et al., J. Appl. Phys. 85, 3222 (1999).

${ }^{14}$ M. Smith et al., Appl. Phys. Lett. 67, 3387 (1995).

${ }^{15}$ B. Santic, C. Merz, U. Kaufmann, R. Niebuhr, H. Obloh, and K. Bachem, Appl. Phys. Lett. 71, 1837 (1997).

${ }^{16}$ M. A. Reshchikov, D. Huang, F. Yun, L. He, H. Morkoç, D. C. Reynolds, S. S. Park, and K. Y. Lee, Appl. Phys. Lett. 79, 3779 (2001).

${ }^{17}$ T. Sogawa, P. V. Santos, S. K. Zhang, S. Eshlaghi, A. D. Wieck, and K. H. Ploog, Phys. Rev. B 63, 121307(R) (2001).
${ }^{18}$ C. Rocke, O. Govorov, A. Wixforth, G. Böhm, and G. Weimann, Phys. Rev. B 57, R6850 (1998).

${ }^{19}$ D. Nelson, B. Gil, M. A. Jacobson, V. D. Kagan, N. Grandjean, B. Beaumont, J. Massies, and P. Gibart, J. Phys.: Condens. Matter 13, 7043 (2001).

${ }^{20}$ T. T. Mnatsakanov, M. E. Levinshtein, L. I. Pomortseva, S. N. Yurkov, G. S. Simin, and M. A. Khan, Solid-State Electron. 47, 111 (2003).

${ }^{21}$ F. Alsina, P. V. Santos, R. Hey, A. García-Cristóbal, and A. Cantarero, Phys. Rev. B 64, 041304(R) (2001).

${ }^{22}$ G. L. Bir and G. E. Pikus, Symmetry and Strain-Induced Effects in Semiconductors (Wiley, New York, 1974).

${ }^{23}$ B. Gil, O. Briot, and R.-L- Aulombard, Phys. Rev. B 52, R17028 (1995).

${ }^{24}$ I. Vurgaftman, J. R. Meyer, and L. R. Ram-Mohan, J. Appl. Phys. 89, 5815 (2001). 